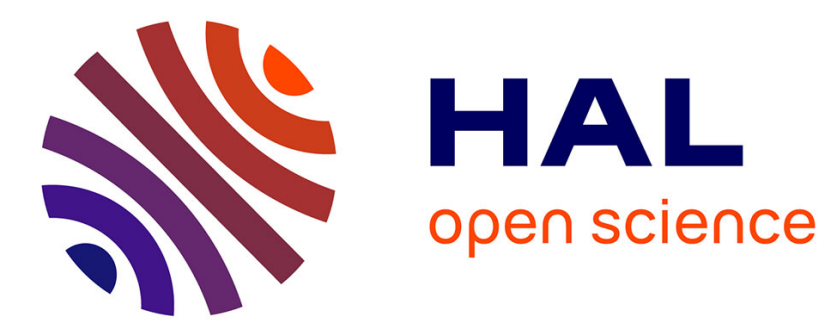

\title{
The Multifaceted Role of Protein Phosphatase 1 in Plasmodium
}

\author{
Jamal Khalife, Aline Fréville, Bénédicte Gnangnon, Christine Pierrot
}

\section{To cite this version:}

Jamal Khalife, Aline Fréville, Bénédicte Gnangnon, Christine Pierrot. The Multifaceted Role of Protein Phosphatase 1 in Plasmodium. Trends in Parasitology, 2021, 37 (2), pp.154-164. 10.1016/j.pt.2020.09.003 . hal-03365541

\section{HAL Id: hal-03365541 \\ https://hal.science/hal-03365541}

Submitted on 5 Oct 2021

HAL is a multi-disciplinary open access archive for the deposit and dissemination of scientific research documents, whether they are published or not. The documents may come from teaching and research institutions in France or abroad, or from public or private research centers.
L'archive ouverte pluridisciplinaire HAL, est destinée au dépôt et à la diffusion de documents scientifiques de niveau recherche, publiés ou non, émanant des établissements d'enseignement et de recherche français ou étrangers, des laboratoires publics ou privés.

\section{(ㅇ)(1) $\$$}

Distributed under a Creative Commons Attribution - NonCommercial - NoDerivatives $\mid 4.0$ 


\section{The multifaceted role of Protein Phosphatase 1 in Plasmodium}

2 Jamal Khalife $^{1 *}$, Aline Fréville ${ }^{1,2, \rho}$, Bénédicte Gnangnon ${ }^{1, \rho}$ and Christine Pierrot ${ }^{1}$ 3

$4 \quad{ }^{1}$ Center for Infection and Immunity of Lille - Biology of Apicomplexan Parasites - UMR

59017 CNRS - U1019 INSERM - Université de Lille - Institut Pasteur de Lille

62 current address: Department of Infection Biology, London School of Tropical Medicine and

7 Hygiene, London, United Kingdom.

8 *Correspondence: jamal.khalife@ pasteur-lille.fr (J. Khalife)

$9 \rho$ : AF and BG equally contributed to this review

10

11 Keywords: Plasmodium, phosphatases, PP1, regulatory subunits, interactome, proteomic 12

13 Abstract

14 Protein Phosphatase type 1 (PP1) forms a wide range of Ser/Thr specific phosphatase 15 holoenzymes which contain one catalytic subunit (PP1c), present in all eukaryotic cells, 16 associated with variable subunits known as regulatory proteins. It has recently been shown that regulators take a leading role in the organization and the control of PP1 functions. Many studies have addressed the role of these regulators in diverse organisms including humans and

19 investigated their link to diseases. In this review we summarize recent advances on PP1c in 20 Plasmodium, its interactome and regulators. As a proof of concept, peptides interfering with 21 the regulator binding capacity of PP1c were shown to inhibit the growth of $P$. falciparum, 22 suggesting their potential as drug precursors. 


\section{Reversible protein phosphorylation: An Overview}

2 The processes of protein phosphorylation and dephosphorylation are involved in most aspects of cell biology by contributing to growth, development and division. These central roles are ensured by protein kinases (see Glossary) and phosphatases that add and remove phosphate groups respectively from serine, threonine and tyrosine residues of proteins in eukaryotes and have a huge impact on start and stop decisions of their functions by affecting structures and shapes. The rapidly acquired and deep understanding of how kinases control diverse cellular functions at the molecular levels and their direct connection to cancer due to mutations, dysregulation or overexpression has brought these enzymes to the forefront of the search for effective inhibitors of kinases for cancer chemotherapy. The progress and development of inhibitors towards kinases have been highlighted in two recent reviews in which most of the FDA-approved inhibitors are discussed $[1,2]$.

The involvement of phosphatases in diverse cellular functions began to emerge through the discovery of natural toxins with inhibitory activities against these enzymes and converging studies using diverse biochemical and biological approaches underline the specific and key roles played by several phosphatases [3]. Among serine/threonine phosphatases, Protein Phosphatase type 1 (PP1) is one of the most broadly and best studied. It is estimated that PP1 accounts for most of dephosphorylation processes with specific activities despite the identity of the central region of its catalytic subunit with other phosphatases [4-6]. PP1 has been shown to be essential in cell cycle progression through its ability to regulate the level of phosphorylation of major mitotic kinases [7-9]. More recent studies established that PP1 loss of function induces termination defects of transcription [10,11]. PP1 holoenzyme formation results from the association of the catalytic subunit (PP1c) with variable partners (designated as PIPs for Phosphatase type 1-Interacting Proteins), known as regulatory subunits, which direct dephosphorylation of hundreds of substrates at the right place and right time [3]. Interestingly, along with the fact that interfering with the function of PP1c is lethal, the deregulation of PP1c/PIPs interactions has been shown to be involved in several human diseases [12]. In consequence, drug targeting of these holoenzymes is now being more carefully interrogated and considered although it is still challenging. A recent study based on the inhibition of a PP1c/PIP interaction characterized a lead that showed efficacy in a mouse model of Huntington's disease [13, 14]. Powerful arguments from mouse genetic studies that support the need to pursue targeting the PP1 holoenzymes have been elegantly reported in a recent review, suggesting that promising ways to develop selective and potent drugs involve the disruption of interactions between PP1c and PIPs [12]. 
1 In this review, we summarize recent advances in the role of phosphatases during the malaria

2 parasite life cycle and focus on PP1c and its PIPs. We also discuss the associated structural 3 and functional networks and potential means to interfere with these functions for medical 4 benefits.

\section{Phosphatases in Plasmodium: Where do we stand?}

Plasmodium species affecting humans are responsible for an estimated 228 million malaria cases worldwide, mainly in Africa where they cause major health, social and economic problems. P. falciparum remains accountable for the greatest part of this burden [15]. A better understanding of Plasmodium biology remains essential to refuel the research strategies and to reveal druggable pathways to tackle the development of this deadly parasite.

Plasmodium has an elaborate and fascinating life cycle to adapt and to survive within different hosts and tissues [16]. It is transmitted back and forth between a vertebrate (intermediate host) and an Anopheles mosquito (definitive host), in which a bottleneck phase is rapidly followed by a period of replication, leading to the generation of thousands of progeny ready to spread the infection. One of the most intriguing features of Plasmodium development is the closed mitosis strategy used for replication, which involves the formation, during schizogony and sporogony, of a syncytium followed by budding and cytokinesis $[17,18]$. Equally interesting is the gametogenesis where three rounds of rapid DNA replication occur within minutes to generate 8 fertile male gametes (Figure 1). As those processes rely on highly flexible, adaptable and parasite-specific mechanisms, they are of great interest for the malaria scientific community aiming to uncover Plasmodium-specific druggable pathways.

Recently, with the massive technological improvement of reverse genetics, multi-omics tools, as well as the availability of databases to speed up data mining, major breakthroughs have been achieved in deciphering the role of phosphatases in the Plasmodium life cycle. Functional screens and characterization of engineered parasite lines led to the identification of crucial phospho-signaling pathways affecting Plasmodium specific development [19-23]. Although some of the phosphatases identified in Plasmodium are conserved across eukaryotic phyla which were reported in a recent review [23], others belong to subfamilies restricted to apicomplexans and plants (Kelch-like domain containing protein phosphatase (PPKL) and Shewanella-like phosphatases (SLP)) or are parasite-specific such as the N-terminus EF-hand motif protein phosphatase (EFPP) [24]. Functional analyses conducted on the unique member 
1 transmission in the mosquito [25, 26]. In Plasmodium berghei ( $\mathrm{Pb}$, a rodent malaria parasite,

2 exploited as a model for $P$. falciparum infection), SLP1 has been shown to be crucial to microneme formation and ookinete development. Ultimately, its deletion resulted in a complete inhibition of oocyst formation [27]. In P. falciparum, SLP2 has been found enclosed in vesicles at the apex of merozoites and was first proposed to be involved in the invasion processes [28] although recent studies suggest that the protein is more likely dispensable during the whole life cycle of both $P$. berghei and $P$. falciparum $[19,29]$.

\section{PfPP1c, cornerstone of dephosphorylation processes}

PP1c emerged after the divergence of Archaea and eukaryotes and was among the first phosphatases biochemically characterized and cloned. It contains a single polypeptide chain with a biologically active domain [30, 31] and was reported as one of the most active phosphatases with its ability to increase the dephosphorylation rate by a factor of $10^{21}$ [32]. PP1c is extremely well conserved as illustrated not only at the protein sequence identity level [33] but also by its capacity to rescue phenotypes linked to a PP1c loss-of-function via crosscomplementation between diverse organisms, including Plasmodium [33-35].

Unlike the human genome, which contains three PPlc genes encoding four isoforms $(\alpha, \beta / \delta$ and $\gamma$ ), the Plasmodium genomes encode only one PP1 catalytic subunit [23]. PfPP1c is $80 \%$ identical ( $88 \%$ similar) to $H s \mathrm{PP} 1 \mathrm{c}$ and its predicted secondary and overall tertiary structures are also well conserved, with $9 \alpha$-helices and $11 \beta$-strands (Figure 2). The catalytic site is located at the surface of the protein as described for $H s$ PP1c [36, 37]. Surprisingly, Plasmodium PP1c lacks a portion of $18 \mathrm{AA}$ at the $\mathrm{Ct}$ side when compared to its human counterpart. This part contains a threonine residue (Thr320) whose phosphorylation is dynamically controlled, playing an extremely important role in the regulation of mammalian PP1c activity and in particular in cell mitosis entry [38, 39]. Hence this type of regulation by PP1c does not seem to take place in Plasmodium. PfPP1c is expressed throughout the parasite Intraerythrocytic Development Cycle (IDC) with an upregulation of its expression from the trophozoite up to the schizont stage. It is localized in the parasite nucleus and cytoplasm [40-42]. This was further confirmed in P. berghei where $P b P P 1 c$ was additionally found to be expressed in sexual and liver stages [19, 43]. PfPP1c has also been localized at Maurer's clefts during the IDC [44]. The major role of PfPP1c in the dephosphorylation processes was first suggested via the use of phosphatase inhibitors, including okadaic acid, calyculin and microcystin [33, 45, 46]. Subsequently, various reverse 
1 in P. falciparum [47] and P. berghei [19, 48]. The use of two inducible knockdown (KD)

2 approaches confirmed the critical role of PfPP1c for the timely regulation of parasite asexual development [41]. The knock down of Pfpplc in rings significantly impaired DNA replication, resulting in the formation of multinucleate schizonts with a reduced number of nuclei and a delayed IDC. At both early or late blood stages, the absence of PfPP1c led to a blockage of egress as exonemes failed to excrete the protease PfSUB1 that orchestrates early stages of egress, and ultimately the rupture of the parasitophorous vacuole membrane [41, 49]. Finally, as microneme discharge is prevented in these parasites, the egress-to-invasion transition was also impaired [41].

To identify potential proteins (de)phosphorylated in a PfPP1c-dependent manner a phosphoproteomic approach has been implemented in parasites in which PfPP1c was knocked down. The analysis revealed hyperphosphorylation of the Ser-29 PfHistone 3 which corresponds to the human ortholog Ser-28, a PP1-targeted site for mitotic exit and metaphase [50, 51]. Additionally, potential substrates as diverse as chromatin factors, AP2 transcription factors or vacuolar-proteins-sorting family (VSP) members have been identified. Although additional investigations are required to confirm the identified substrates, the analysis pointed out some exciting candidates such as PfHECT1 or GCa (guanylyl cyclase alpha). PfHECT1 is an uncharacterized protein containing a C-terminal HECT E3 protein-ubiquitin ligase domain which had been previously identified in silico as a potential PfPIP [20]. PfGCa, on the other hand, is a well-known effector essential for cGMP production and stimulation of PfPKG, a critical component of egress required for PfSUB1 discharge from exonemes [52-54]. Using a chemical-genetics assay, the authors demonstrated the essential ability of PfPP1c to regulate these proteins and their egress-related activity. They also revealed the involvement of host serum phospholipid PtdC (phosphatidylcholine) through the dephosphorylation by PfPP1c of a phospholipid transporter domain situated at the N-terminal of GCa. In sum, the authors proposed a model where PfPP1c is a central regulator for egress, essential for balancing environmental signals and intra-cellular pathways to ensure timely and proper parasite propagation in the blood [41]. An additional conditional KD study conducted in P. berghei showed that $P b P P 1 c$ did not play any important role in sporozoites and liver stages, despite being expressed, but seemed critical for the establishment of blood infection after pplcKD sporozoite injection in mice [43]. Since the $p p l$ genomic locus was specifically disrupted when the parasites were developing into sporozoites, no information can thus be drawn about upstream development occurring in mosquitoes. 


\section{Regulatory subunits: Spatiotemporal orientation of the function of PP1c}

2 Converging evidence from numerous studies strongly supports the model that the PP1c

3 platform acts in a highly and regulated manner through its combinatorial capacity to interact with many PIPs. PIPs are structurally unrelated and expressed at key points during the cell cycle. They can thus orient the "dephosphorylation power" of PP1c in time and space, by regulating the localization and/or the activity of the enzyme [55, 56]. PIPs bind the phosphatase surface grooves via docking motifs including the $\mathrm{RVxF}$ motif, which was originally the best-defined binding site for PP1, along with other motifs (Box 1) [57, 58]. Also, ensuring the functional diversity of PP1 holoenzymes towards substrates prevents PP1c from being deleterious to the cell [59, 60]. In vertebrates, at least 200 PIPs have been identified [61] while it has been estimated that as many as 650 distinct PP1 complexes may exist [62].

\section{Conserved Plasmodium PIPs reveal parasite specific features}

Despite the essential role of PfPP1c, little was initially known about its regulation in Plasmodium. Based on comparative sequence analyses, our earlier work revealed that $P$. falciparum expresses only four well-known conserved PfPP1c-regulators described in mammals: PfLRR1 (Leucine Rich repeat 1), PfI2 (Inhibitor 2), PfI3 (Inhibitor 3), and Pfeif2ß (eukaryotic initiation factor 2B) [40, 63-65]. At the functional level, several lines of evidence have indicated that yeast or human SDS22 (PfLRR1 orthologs) and inhibitor 3, known as PP1 inhibitors in vitro [66], are involved in a common pathway regulating cell division by stabilizing kinetochore spindle attachment and proper chromosome segregation [67-71]. Regarding Inhibitor 2, its implication in the regulation of mitosis, and more specifically in cytokinesis, has been reported [72, 73]. Interestingly, a recent proteomic approach aiming to explore PfPP1c signaling events revealed a significant accumulation of PfLRR1 as well as $P f 12$ in a P. falciparum strain in which PP1c was depleted [41]. The functional significance of such an accumulation on the progress of IDC is still unknown. A targeted knock out approach suggested the essentiality of the 4 PfPIPs during its IDC [40, 63-65]. However, a more recent study based on saturation mutagenesis aiming to identify $P$. falciparum essential genes nuanced these results, as it suggested that $P f \mathrm{I} 2$ and $P f \mathrm{I} 3$ are non-essential during IDC. It is, however, worth noting that PfI2 may have a function during blood stage development as insertions in its gene were associated with a slow growth phenotype [47].

33 Biochemical and structure-activity relationship studies revealed the ability of PfLRR1, PfI2, 
1 of PP1c-PfLRR1 binding, pepscan analysis revealed that only one synthetic peptide derived 2 from Leucine-Rich Repeats (LRRs) was involved in this binding [74]. Unexpectedly, the 3 peptide corresponding to the C-terminal LRR cap domain, previously known as crucial for the stabilization and integrity of the protein [75], was also shown to directly interact with PP1c [74]. At this point, it is important to emphasize that a recent work on its human ortholog, SDS22, showed a different pattern of binding which involves six LRR motifs, without reporting a role of the C-terminal LRR cap domain [76]. In Plasmodium, this needs further work on the structural interactions and raises an interesting question about the role of the PfLRR1 cap domain on the function of PP1c.

With respect to $P f 12, P f 13$, and $P f$ eif $2 \beta$, their primary amino acid sequences did show the presence of $\mathrm{RVxF}$ consensus motifs. As expected, biochemical and mutational analysis showed a main contribution of this motif to PfPP1c binding. The 3D structures of PfI2 and PfI3 have been solved by NMR spectroscopy alone and in the presence of PfPP1c, confirming the interaction via the RVxF motif in solution. Interestingly, Pfl2 presents a more complex interaction pattern which involves an additional FxxR/KxR/K (also referred to in this paper as Fxx) motif also described in PP1c binding anti-apoptotic proteins and ion transporters [7779]. Divergences were also observed in $P f I 2$ at the primary sequence level when compared to its human ortholog in which the $\mathrm{RVxF}$ considerably deviates from the consensus sequence. The protein is also around $30 \%$ shorter than its mammalian ortholog and does not possess a SILK motif, which has been showed to play a critical role in both PP1c binding and regulation in mammals $[57,80]$. Based on these observations, the mode of binding of PfI2 to PfPP1c differs from its human ortholog. Also, it should be noted that the nature of the $\mathrm{x}$ residue in the $\mathrm{RVxF}$ motif could play a role in the stabilization of the interaction as previously reported [81]. This x residue is not conserved between $P$. falciparum conserved PIPs and their human counterparts. More importantly, in the case of $P f \mathrm{I} 3$, despite its sequence similarity with its yeast and human orthologs, it seems to have a distinct regulatory role on PfPP1c as attested by a stimulation rather than an inhibition of the phosphatase activity [64]. All these differences in the binding and regulation of PP1c highlight some intriguing specific characteristics of Plasmodium PP1 holoenzymes.

\section{Insights into Plasmodium specific PIPs}

Plasmodium PP1c interactome -. To decipher the signaling pathways regulated by this phosphatase, the Plasmodium PP1c interactome has been explored using different and 
1 RVxF motif screenings, reported 134 and 55 PfPIPs respectively [20]. Additionally, a recent 2 proteomics study carried out in $P$. berghei schizonts led to the identification of 178 potential 3 PIPs [82] among which 19 proteins have been already shown to physically interact with PP1c either by $\mathrm{Y} 2 \mathrm{H}$ approach and/or binding assays using recombinant proteins in $P$. falciparum (supplemental Table S1) [20, 40, 63]. Strikingly, 80 PIPs (i.e. 70\% of the proteins for which data are available) may be essential for $P$. berghei IDC completion, as assessed by the lack of viable knockout parasites [48] (Figure 3A). More details of the phenotypes of the potential $P b$ PIPs are depicted in Figure 1 and supplemental Table S2. A GO term analysis of $P b P I P S$ showed significant enrichment of distinct biological pathways (mostly proteasome assembly, protein folding and translational processes) which confirms and extends to Plasmodium the diverse functions of the PP1 holoenzyme (supplemental Table S3).

Comparative studies revealed that $31.5 \%$ of the potential PbPIPs do not have homologs in human and thus can be considered as promising drug targets if the binding with the phosphatase can be confirmed. In this context, two proteins previously identified as PfPIPs in $\mathrm{Y} 2 \mathrm{H}$ screening whose expression is restricted to Plasmodium were further investigated. Biochemical and reverse genetics approaches determined that the first, designated RCC-PIP, can bind/transport not only PfPP1c but also the kinase PfCDPK7 [83]. The second PIP, $P f$ GEXP15, is a regulator that increases PfPP1c activity [82]. The KO line of its $P$. berghei ortholog failed to induce hyper-parasitemia and experimental cerebral malaria. The Pbgexp15-KO line was also unable to produce oocysts in mosquitoes, highlighting once again the role of Plasmodium PIPs in both asexual and sexual parasite stages.

Potential Plasmodium-specific PP1c regulators and substrates - Assuming that the direct and physical interaction of regulators to PP1c is crucial for their functions, it was therefore necessary to examine their mode of binding. In this context, it is interesting to note that 93 $P b P I P s$ identified from the IP/MS interactome [82] have an RVxF motif defined by Wakula et al. [84] (Box 1), with 16 of them fitting with the less sensitive but more specific consensus sequence defined by Meiselbach et al. [85]. Enrichment analysis showed that the RVxF motif conforming to the Meiselbach sequence was significantly enriched (fold enrichment: 1.86, hypergeometric P-value: 0.01). Fifty-one PIPs had at least one additional interaction motif (SILK, Fxx) (Figure 3B, Box 1). Further analysis showed that 14 PIPs have a highly specific $\mathrm{RVxF}$ motif [85] and at least one secondary interaction motif, and two PIPs show a less specific RVxF motif [84] together with a Fxx and a SILK motif (supplemental Table S4). 
1 activity. Overall, this provides insights into the formation of multiple PP1 complexes in

2 Plasmodium and explains why PP1 can be associated with several signaling pathways.

3 It has been previously reported that several vertebrate PIPs are also PP1 substrates [62]. In an

4 attempt to find out potential Plasmodium PP1c substrates among the PIPs identified above,

5 the phosphorylation status of their $P$. falciparum orthologs was examined from

6 phosphoproteomics data collected in Pfpplc-knockdown parasites [41]. This led to the

7 identification of 4 PIPs showing an increase in their level of phosphorylation following

8 PfPP1c depletion (supplemental Table S5). Furthermore, two of them possess three PP1c

9 binding motifs (supplemental Table S4), suggesting that they could belong to a regulator/substrate group.

PP1c complexes as target for the design of innovative of anti-malaria peptides - The above analyses define an emerging and robust theme in the Plasmodium PP1 field, where PP1c forms complexes with diverse (regulatory) PIPs whose interactions are crucial for the correct functions of this enzyme during the lifecycle of blood stage parasites. From this point of view, it is rational to attempt to interfere with these interactions and evaluate how this impacts parasite growth. Synthetic peptides corresponding to the RVxF motifs of PfI2 and PfI3, able to bind PfPP1c in vitro, successfully induced a drastic inhibition of P. falciparum growth in vitro [63] while peptide corresponding to Fxx motif of $P f \mathrm{I} 2$ showed slight but significant growth inhibition [65, 78]. In a more recent study, the peptides derived from PfLRR1 revealed the capacity of one peptide to inhibit both blood stage parasite growth and SPZ development in hepatocytes [74]. Together, these results provide proof of concept that the PP1c/PIP interfaces are relevant targets for the design of new antimalarials. This could be achieved via the development of specific small molecules that mimic the primary or secondary PP1c docking motifs.

\section{Concluding Remarks}

The idea that PP1 is a nonspecific housekeeping phosphatase, which was initially based on in vitro data obtained with its catalytic subunit PP1c, is no longer tenable. The high combinatorial capacity of the catalytic subunit with hundreds of PIPs makes it a highly regulated and specific phosphatase. From Plasmodium PP1c interactome studies, there are many distinct complexes with restricted substrate specificity known to be involved in major and essential pathways in Plasmodium. Despite the recent advances, more in-depth analyses are required to understand the multifaceted role of PP1 holoenzymes during Plasmodium life 
1 cycle, as well as in other Apicomplexa (see outstanding questions). In a short-term outlook, 2 obtaining structural data on the interaction of PP1c with PIPs will reveal how PP1 can be 3 efficiently targeted with small molecules. In a long-term outlook, it will become important to determine the activity of various PP1c-PIP complexes toward different substrates, including those which will be identified in Plasmodium. This will help defining specific molecular mechanisms and pathways. This might in turn contribute to the elaboration of innovative ways to control parasite development, as has been shown via the use of interfering peptides that inhibit $P$. falciparum growth.

ACKNOWLEDGMENTS: We would like to thank Dr Raymond J Pierce for his useful discussions and critical reading of the manuscript and "La region Hauts-de-France" for funding AF (“Accueil de Jeunes Chercheurs” award, 2017-2019).

\section{References}

1. Kannaiyan, R. and Mahadevan, D. (2018) A comprehensive review of protein kinase inhibitors for cancer therapy. Expert Rev Anticancer Ther 18, 1249-1270 2. Roskoski, R. (2019) Properties of FDA-approved small molecule protein kinase inhibitors. Pharmacol Res 144, 19-50

3. Brautigan, D.L. and Shenolikar, S. (2018) Protein Serine/Threonine Phosphatases: Keys to Unlocking Regulators and Substrates. Аnпи Rev Biochem 87, 921-964

4. Cohen, P. (1989) The Structure and Regulation of Protein Phosphatases. Annu Rev Biochem 58, 453-508

5. Virshup, D.M. and Shenolikar, S. (2009) From Promiscuity to Precision: Protein Phosphatases Get a Makeover. Mol Cell 33, 537-545

6. MacDougall, L.K., et al. (1991) Identification of the major protein phosphatases in mammalian cardiac muscle which dephosphorylate phospholamban. Eur J Biochem 196, 725-734 7. Nasa, I. and Kettenbach, A.N. (2018) Coordination of Protein Kinase and Phosphoprotein Phosphatase Activities in Mitosis. Front Cell Dev Biol 6 8. Ramos, F., et al. (2019) Role of protein phosphatases PP1, PP2A, PP4 and Cdc14 in the DNA damage response. Cell stress 3, 70-85

31 9. Kinoshita, N., et al. (1990) Distinct, essential roles of type 1 and 2A protein phosphatases in the 32 control of the fission yeast cell division cycle. Cell 63, 405-415

33 10. Cossa, G., et al. (2020) Localized Inhibition of Protein Phosphatase 1 by NUAK1 Promotes

34 Spliceosome Activity and Reveals a MYC-Sensitive Feedback Control of Transcription. Mol Cell 77, 1322-1339.e1311 
1 11. Kecman, T., et al. (2018) Elongation/Termination Factor Exchange Mediated by PP1

2 Phosphatase Orchestrates Transcription Termination. Cell Rep 25, 259-269.e255

3 12. Ferreira, M., et al. (2019) Functions and therapeutic potential of protein phosphatase 1: Insights

4 from mouse genetics. BBA-Mol Cell Res 1866, 16-30

5 13. Krzyzosiak, A., et al. (2018) Target-Based Discovery of an Inhibitor of the Regulatory 6 Phosphatase PPP1R15B. Cell 174, 1216-1228.e1219

7 14. Vagnarelli, P. and Alessi, D.R. (2018) PP1 Phosphatase Complexes: Undruggable No Longer.

8 Cell 174, 1049-1051

9 15. World Health, O. (2019) World malaria report 2019. World Health Organization

10 16. Striepen, B., et al. (2007) Building the perfect parasite: cell division in apicomplexa. PLoS 11 Pathog 3, e78-e78

12 17. Francia, M.E. and Striepen, B. (2014) Cell division in apicomplexan parasites. Nat Rev Microbiol $13 \quad 12,125-136$

14 18. Matthews, H., et al. (2018) Checks and balances? DNA replication and the cell cycle in Plasmodium. Parasites \& vectors 11, 216-216

19. Guttery, D.S., et al. (2014) Genome-wide functional analysis of Plasmodium protein phosphatases reveals key regulators of parasite development and differentiation. Cell Host Microbe $16,128-140$

20. Hollin, T., et al. (2016) Analysis of the interactome of the Ser/Thr Protein Phosphatase type 1 in Plasmodium falciparum. BMC Genomics 17, 246

21. Pandey, R., et al. (2014) Genome wide in silico analysis of Plasmodium falciparum phosphatome. BMC Genomics 15, 1024

22. Carvalho, T.G., et al. (2016) The ins and outs of phosphosignalling in Plasmodium: Parasite regulation and host cell manipulation. Mol Biochem Parasitol 208, 2-15 23. Yang, C. and Arrizabalaga, G. (2017) The serine/threonine phosphatases of apicomplexan parasites. Mol Microbiol 106, 1-21

24. Kutuzov, M.A. and Andreeva, A.V. (2008) Protein Ser/Thr phosphatases of parasitic protozoa. Mol Biochem Parasitol 161, 81-90

25. Guttery, D.S., et al. (2012) A unique protein phosphatase with kelch-like domains (PPKL) in Plasmodium modulates ookinete differentiation, motility and invasion. PLoS Pathog 8, e1002948

26. Philip, N., et al. (2012) A unique Kelch domain phosphatase in Plasmodium regulates ookinete morphology, motility and invasion. PLOS ONE 7, e44617 27. Patzewitz, E.M., et al. (2013) An ancient protein phosphatase, SHLP1, is critical to microneme development in Plasmodium ookinetes and parasite transmission. Cell Rep 3, $622-629$ 28. Fernandez-Pol, S., et al. (2013) A bacterial phosphatase-like enzyme of the malaria parasite Plasmodium falciparum possesses tyrosine phosphatase activity and is implicated in the regulation of band 3 dynamics during parasite invasion. Eukaryot Cell 12, 1179-1191 
1 29. Miliu, A., et al. (2017) Shelph2, a bacterial-like phosphatase of the malaria parasite Plasmodium

2 falciparum, is dispensable during asexual blood stage. PLOS ONE 12, e0187073

3 30. Lee, E.Y., et al. (1999) Phosphorylase phosphatase: new horizons for an old enzyme. Front 4 Biosci 4, D270-285

5 31. Berndt, N., et al. (1987) Isolation and sequence analysis of a cDNA clone encoding a type-1 protein phosphatase catalytic subunit: Homology with protein phosphatase 2A. FEBS Letters 223, $340-346$

32. Lad, C., et al. (2003) The rate of hydrolysis of phosphomonoester dianions and the exceptional catalytic proficiencies of protein and inositol phosphatases. Proc Natl Acad Sci U S A 100, 5607-5610 33. Bhattacharyya, M.K., et al. (2002) Plasmodium falciparum protein phosphatase type 1 functionally complements a glc7 mutant in Saccharomyces cerevisiae. Int J Parasitol 32, 739-747 34. Doonan, J.H., et al. (1991) A cDNA encoding rabbit muscle protein phosphatase 1 alpha complements the Aspergillus cell cycle mutation, bimG11. J Biol Chem 266, 18889-18894 35. Gibbons, J.A., et al. (2007) Expression of Human Protein Phosphatase-1 in Saccharomyces cerevisiae Highlights the Role of Phosphatase Isoforms in Regulating Eukaryotic Functions. $J$ Biol Chem 282, 21838-21847

36. Egloff, M.P., et al. (1995) Crystal structure of the catalytic subunit of human protein phosphatase 1 and its complex with tungstate. J Mol Biol 254, 942-959

37. Egloff, M.P., et al. (1997) Structural basis for the recognition of regulatory subunits by the catalytic subunit of protein phosphatase 1. Embo J 16, 1876-1887

38. Dohadwala, M., et al. (1994) Phosphorylation and inactivation of protein phosphatase 1 by cyclin-dependent kinases. Proc Natl Acad Sci U S A 91, 6408-6412

39. Kwon, Y.G., et al. (1997) Cell cycle-dependent phosphorylation of mammalian protein phosphatase 1 by cdc2 kinase. Proc Natl Acad Sci U S A 94, 2168-2173

40. Daher, W., et al. (2006) Regulation of protein phosphatase type 1 and cell cycle progression by PfLRR1, a novel leucine-rich repeat protein of the human malaria parasite Plasmodium falciparum. Mol Microbiol 60, 578-590

41. Paul, A.S., et al. (2020) Co-option of Plasmodium falciparum PP1 for egress from host erythrocytes. Nat Commun 11, 3532

42. Howick, V.M., et al. (2019) The Malaria Cell Atlas: Single parasite transcriptomes across the complete Plasmodium life cycle. Science 365, eaaw2619 43. Zhang, M., et al. (2016) UIS2: A Unique Phosphatase Required for the Development of Plasmodium Liver Stages. PLoS Pathog 12, e1005370

44. Blisnick, T., et al. (2006) Protein phosphatase 1, a Plasmodium falciparum essential enzyme, is exported to the host cell and implicated in the release of infectious merozoites. Cell Microbiol 8, 591- 
1 45. Dombradi, V., et al. (2004) Protein Phosphatase 1. In Protein Phosphatases (Arino, J. and 2 Alexander, D., eds), Springer-Verlag Berlin Heidelberg

3 46. Yokoyama, D., et al. (1998) Modulation of the growth of Plasmodium falciparum in vitro by 4 protein serine/threonine phosphatase inhibitors. Biochem Biophys Res Commun 247, 18-23

5 47. Zhang, M., et al. (2018) Uncovering the essential genes of the human malaria parasite 6 Plasmodium falciparum by saturation mutagenesis. Science 360

7 48. Bushell, E., et al. (2017) Functional Profiling of a Plasmodium Genome Reveals an Abundance 8 of Essential Genes. Cell 170, 260-272 e268

9 49. Collins, C.R., et al. (2017) The Plasmodium falciparum pseudoprotease SERA5 regulates the 10 kinetics and efficiency of malaria parasite egress from host erythrocytes. PLoS Pathog 13, e1006453$11 \mathrm{e} 1006453$

12 50. Goto, H., et al. (2002) Aurora-B phosphorylates Histone H3 at serine28 with regard to the mitotic 13 chromosome condensation. Genes Cells 7, 11-17

14 51. de Castro, I.J., et al. (2017) Network of phosphatases and HDAC complexes at repressed 15 chromatin. Cell Cycle 16, 2011-2017

16 52. Taylor, H.M., et al. (2010) The malaria parasite cyclic GMP-dependent protein kinase plays a 17 central role in blood-stage schizogony. Eukaryot Cell 9, 37-45

18 53. Collins, C.R., et al. (2013) Malaria parasite cGMP-dependent protein kinase regulates blood stage merozoite secretory organelle discharge and egress. PLoS Pathog 9, e1003344-e1003344

54. Carucci, D.J., et al. (2000) Guanylyl Cyclase Activity Associated with Putative Bifunctional Integral Membrane Proteins in Plasmodium falciparum. J Biol Chem 275, 22147-22156 55. Ceulemans, H. and Bollen, M. (2004) Functional diversity of protein phosphatase-1, a cellular economizer and reset button. Physiol Rev 84, 1-39 56. Ceulemans, H., et al. (2002) Binding of the concave surface of the Sds22 superhelix to the alpha 4/alpha 5/alpha 6-triangle of protein phosphatase-1. J Biol Chem 277, 47331-47337 57. Hurley, T.D., et al. (2007) Structural basis for regulation of protein phosphatase 1 by inhibitor-2. J Biol Chem 282, 28874-28883

58. Terrak, M., et al. (2004) Structural basis of protein phosphatase 1 regulation. Nature 429, 780784

59. Chatterjee, J., et al. (2012) Development of a peptide that selectively activates protein phosphatase-1 in living cells. Angew Chem Int Ed Engl 51, 10054-10059 60. Verbinnen, I., et al. (2017) Biogenesis and activity regulation of protein phosphatase 1. Biochem Soc Trans 45, 89-99 61. Heroes, E., et al. (2013) The PP1 binding code: a molecular-lego strategy that governs specificity. Febs J 280, 584-595 
1 63. Freville, A., et al. (2013) Plasmodium falciparum encodes a conserved active inhibitor-2 for

2 Protein Phosphatase type 1: perspectives for novel anti-plasmodial therapy. BMC Biol 11, 80 64. Freville, A., et al. (2012) Plasmodium falciparum inhibitor-3 homolog increases protein phosphatase type 1 activity and is essential for parasitic survival. J Biol Chem 287, 1306-1321 65. Tellier, G., et al. (2016) Identification of Plasmodium falciparum Translation Initiation eIF2beta Subunit: Direct Interaction with Protein Phosphatase Type 1. Front Microbiol 7, 777 66. Lesage, B., et al. (2007) A complex of catalytically inactive protein phosphatase-1 sandwiched between Sds22 and inhibitor-3. Biochemistry 46, 8909-8919 67. Bharucha, J.P., et al. (2008) Ypi1, a positive regulator of nuclear protein phosphatase type 1 activity in Saccharomyces cerevisiae. Mol Biol Cell 19, 1032-1045 68. Eiteneuer, A., et al. (2014) Inhibitor-3 ensures bipolar mitotic spindle attachment by limiting association of SDS22 with kinetochore-bound protein phosphatase-1. Embo J 33, 2704-2720 69. Garcia-Gimeno, M.A., et al. (2003) Molecular characterization of Ypi1, a novel Saccharomyces cerevisiae type 1 protein phosphatase inhibitor. J Biol Chem 278, 47744-47752 70. Pedelini, L., et al. (2007) YPI1 and SDS22 proteins regulate the nuclear localization and function of yeast type 1 phosphatase Glc7. J Biol Chem 282, 3282-3292 71. Peggie, M.W., et al. (2002) Essential functions of Sds22p in chromosome stability and nuclear localization of PP1. J Cell Sci 115, 195-206 72. Brautigan, D.L., et al. (1990) Cell cycle oscillation of phosphatase inhibitor-2 in rat fibroblasts coincident with p34cdc2 restriction. Nature 344, 74-78 73. Wang, W., et al. (2008) Phosphatase inhibitor-2 balances protein phosphatase 1 and aurora B kinase for chromosome segregation and cytokinesis in human retinal epithelial cells. Mol Biol Cell 19, $4852-4862$

74. Pierrot, C., et al. (2018) Peptides derived from Plasmodium falciparum leucine-rich repeat 1 bind to serine/threonine phosphatase type 1 and inhibit parasite growth in vitro. Drug Des Devel Ther 12, $85-88$

75. Dao, T.P., et al. (2014) Capping motifs stabilize the leucine-rich repeat protein PP32 and rigidify adjacent repeats. Protein Sci 23, 801-811

76. Heroes, E., et al. (2019) Structure-Guided Exploration of SDS22 Interactions with Protein Phosphatase PP1 and the Splicing Factor BCLAF1. Structure 27, 507-518 e505 77. Ayllon, V., et al. (2002) The anti-apoptotic molecules Bcl-xL and Bcl-w target protein phosphatase 1alpha to Bad. Eur J Immunol 32, 1847-1855 78. Freville, A., et al. (2014) Identification of a Plasmodium falciparum inhibitor-2 motif involved in the binding and regulation activity of protein phosphatase type 1. Febs $J$ 281, 4519-4534 79. Lee, K.P., et al. (2018) Functional identification of protein phosphatase 1-binding consensus 
1 80. Yang, J., et al. (2000) Interaction of inhibitor-2 with the catalytic subunit of type 1 protein 2 phosphatase. Identification of a sequence analogous to the consensus type 1 protein phosphatase-

3 binding motif. J Biol Chem 275, 22635-22644

4 81. Ragusa, M.J., et al. (2010) Spinophilin directs protein phosphatase 1 specificity by blocking 5 substrate binding sites. Nat Struct Mol Biol 17, 459-464 82. Hollin, T., et al. (2019) Essential role of GEXP15, a specific Protein Phosphatase type 1 partner, in Plasmodium berghei in asexual erythrocytic proliferation and transmission. PLoS Pathog 15, $8 \quad \mathrm{e} 1007973$ 83. Lenne, A., et al. (2018) Characterization of a Protein Phosphatase Type-1 and a Kinase Anchoring Protein in Plasmodium falciparum. Front Microbiol 9, 2617-2617 84. Wakula, P., et al. (2003) Degeneracy and function of the ubiquitous RVXF motif that mediates binding to protein phosphatase-1. J Biol Chem 278, 18817-18823 85. Meiselbach, H., et al. (2006) Structural analysis of the protein phosphatase 1 docking motif: molecular description of binding specificities identifies interacting proteins. Chem Biol 13, 49-59 86. Bollen, M. (2001) Combinatorial control of protein phosphatase-1. Trends Biochem Sci 26, 426431

87. Hendrickx, A., et al. (2009) Docking motif-guided mapping of the interactome of protein phosphatase-1. Chem Biol 16, 365-371

\section{Box 1. Structural basis of interaction with PP1c}

- The PP1 binding code is specific, universal, degenerate, overlapping, non-exclusive and dynamic $[60,61]$ :

- Specific: PIPs contain a combination of multiple binding motifs, and thus bind PP1c in a specific way.

- Universal: given the high degree of conservation of PP1c.

- Degenerate: binding motifs come in many sequence variants, which bind PP1c with a given affinity.

- Overlapping: different binding motifs can target the same site on PP1c (but 2 variants cannot bind at the same time).

- Non-exclusive: more than one PIP can bind PP1c simultaneously (as long as they have one non-shared binding motif). 
- Dynamic: the competition between PIPs and PP1c, as well the post-translational modification of binding motifs [7], can impact the formation of holoenzymes.

- The RVxF motif is the most widely occurring binding motif in PIPs

PIPs can be PP1c regulators, substrates, or both. $70 \%$ of PIPs possess an RVxF motif, which makes it the main binding site to PP1c [60, 62]:

- The RVxF motif serves as an anchor for the initial binding of the PIP (it is therefore a primary binding site). It brings the PIP close to PP1c and facilitates binding of secondary interaction sites with lower binding affinity which may in turn modulate the enzymatic activity and/or substrate specificity of the phosphatase [60, 86, 87].

- Table I: Overview of RVxF consensus sequences

\begin{tabular}{|c|c|}
\hline $\begin{array}{l}\text { Wakula RVxF consensus sequence: } \\
{[\mathrm{RK}] \mathrm{X}_{0-1}[\mathrm{VI}]\{\mathrm{P}\}[\mathrm{FW}]}\end{array}$ & $\begin{array}{l}\text { High sensitivity (recapitulates } 90 \% \text { of } \\
\text { existing RVxF motifs) but low specificity } \\
\text { (randomly present in } 25 \% \text { of proteins) [84]. }\end{array}$ \\
\hline Meiselbach $R V x F$ consensus sequence: & sitivity (result of $t$ \\
\hline $\begin{array}{c}\begin{array}{c}\text { These basic residues are } \\
\text { the first anchor point of } \\
\text { the motif to PP1c }\end{array} \\
\begin{array}{r}\text { Nonpolar aromatic residues } \\
\text { are critical at this position } \\
\text { for maximal affinity }\end{array} \\
\text { [HRKD [ACHKMNQRSTV] [VDD [CHKNQRST] FWD } \\
\text { This residue is preterentially a Valine } \\
\text { to bind a hydrophobic pocket on PP1c }\end{array}$ & $\begin{array}{l}\text { mutagenesis analysis to improve our } \\
\text { understanding of the function of each } \\
\text { residues) but its specificity is } \mathbf{1 0} \text { times } \\
\text { Wakula sequence's [85]. }\end{array}$ \\
\hline $\begin{array}{c}\text { Hendrickx RVxF binding mode: } \\
{\left[\mathrm{K}_{54} \mathrm{R}_{34} \mathrm{~L}_{4}\right]\left[\mathrm{K}_{28} \mathrm{R}_{26} \mathrm{~S}_{10} \mathrm{~T}_{9} \mathrm{~A}_{8} \mathrm{M}_{3} \mathrm{~V}_{3} \mathrm{H}_{4} \mathrm{~N}_{3}\right]} \\
{\left[\mathrm{V}_{94} \mathrm{I}_{6}\right]\{\mathrm{FIMYDP}\}\left[\mathrm{F}_{83} \mathrm{~W}_{17}\right]}\end{array}$ & $\begin{array}{l}\text { Combination of the above sequences. Both } \\
\text { high sensitivity and specificity [87]. }\end{array}$ \\
\hline \multicolumn{2}{|c|}{$\begin{array}{l}\text { Caption } \\
{[\mathrm{HRK}]=\text { either Histidine, Arginine or Lysine at this position in the motif }} \\
\mathrm{X}=\text { any residue at this position in the motif } \\
\{\mathrm{P}\}=\text { any residue but Proline at this position in the motif }\end{array}$} \\
\hline
\end{tabular}

- -Examples of secondary binding motifs (i.e. that bind PP1c after a primary binding site like $\mathrm{RVxF}$ anchored the protein partner to the phosphatase) include SILK (consensus sequence [GS]IL[KR]\{DE\}) [57], myosin phosphatase N-terminal element (MyPhoNE consensus sequence RxxQ[VIL][KR]x[YW]) [58, 87], Fxx (consensus sequence Fxx[RK]x[RK]), and HYNE. 
2 Figure Legends

4 Figure 1. Schematic illustration of Plasmodium life cycle showing proteins with known functions identified as potential PIPs in P. berghei. Plasmodium is transmitted between its intermediate and definitive hosts via mosquito bites. Sporozoites are injected in the skin of intermediate hosts by infectious mosquitoes. Some reach the bloodstream and, ultimately, the liver where they will infect hepatocytes. After growth and replication (hepatic schizogony) in the liver, merosomes (merozoite stages contained in the hepatocyte membrane) migrate to the bloodstream. Thousands of merozoites are released upon merosome lysis. These stages are only infectious to erythrocytes. Upon invasion, each merozoite gives a ring which will then mature into a trophozoite and then to a schizont. Each schizont can divide into about 16 merozoites which will be released in the bloodstream upon erythrocyte lysis, thus inducing/sustaining an intra-erythrocytic cycle that will lead to a rise in parasitemia and malaria symptoms. A portion of trophozoites can also differentiate into transmissible stages, called gametocytes. These sexual stages are activated in the mosquito midgut into gametes. Following fertilization, the zygote differentiates into an ookinete able to cross the midgut barrier and develop there into an oocyst. Upon maturation, oocysts give hundreds of sporozoites which will migrate to the mosquito salivary glands, thus allowing them to be easily transmitted to the next intermediate host via mosquito bites. The stage specific function of Plasmodium berghei PIPs are also presented (see supplemental Table S2 for details and references). This scheme has been created using PowerPoint from Microsoft Office 365.

Figure 2. The high level of conservation of PP1c between Plasmodium and Human. Since human PP1c (HsPP1c) has never been entirely crystallized (i.e. available crystal structures do not cover the last 30 AA of the protein), its tertiary structure was predicted using I-Tasser (https://zhanglab.ccmb.med.umich.edu/I-TASSER/). The model for PfPP1c tertiary structure was uploaded from

SwissModel (https://swissmodel.expasy.org/repository/uniprot/Q8ILV1?csm=9CE0B7C29FEFEFC6). Both models were superimposed using the MatchMaker tool from Chimera (version 1.14). This superimposition illustrates the high level of conservation between the two proteins, except for their C-terminal extremities. HsPP1c and PfPP1c are shown in brown and blue respectively; the C-terminal extremity of $H_{s} \mathrm{PP} 1 \mathrm{c}$ has been circled in purple. 
2 Figure 3. Feature analysis of the PIPs identified in P. berghei schizonts. A. Pie chart of the 3 essentiality of the PIPs in P. berghei for the parasite IDC, as determined in the PlasmoGEM 4 study [48]. Essential, Dispensable and Slow are for relative growth rate of 0.1, 1.0 and 5 between 0.1 and 1.0 respectively. B. Venn diagram of the distribution of $P$. berghei PIPs 6 according to their PP1c binding motifs. RVxF_W and RVxF_M stand for RVxF consensus 7 sequences as defined by Wakula et al [84] and Meiselbalch et al [85] respectively and Fxx 8 stands for FxxR/KxxR/K motif. The consensus sequences of binding motifs are detailed in 9 Box 1. This diagram has been created using $\mathrm{R}$ version 3.6.3 and the eulerr package. 10 11 12 13 